\title{
Agriculture urbaine et périurbaine (AUP) et économie des ménages agri-urbains à Dakar (Sénégal)
}

\author{
Abou BA ${ }^{* 1}$, Nicola Cantoreggi $\mathrm{PhD}^{2}$ \\ ${ }^{1}$ Doctorant, Institut des sciences de l'environnement (ISE), Université de Genève, \\ ${ }^{2}$ Chargé de cours, Institut des sciences de l'environnement, Université de Genève,
}

\begin{abstract}
Résumé-Les dynamiques actuelles de l'urbanisation en Afrique sub-saharienne posent d'importants défis en matière d'alimentation et de sécurité alimentaire des populations urbaines. Dans ce contexte, la réflexion sur la fonction alimentaire de l'agriculture urbaine et périurbaine (AUP) trouve toute sa pertinence et de nombreux travaux ont été consacrés à l'analyse de la fonction alimentaire de l'AUP. Par contre, peu de recherches ont porté sur la dimension essentiellement commerciale de l'activité, permettant de générer des revenus susceptibles de répondre aux différents besoins, alimentaires et non-alimentaires, des ménages agri-urbains. La présente contribution s'intéresse à cet aspect particulier de l'activité maraichère pratiquée dans la région de Dakar dans trois zones de production impliquant 214 maraîchers.

Les résultats obtenus montrent que l'AUP à travers le maraîchage urbain à Dakar constitue une activité commerciale à part entière dont les revenus monétaires sont de loin plus importants que le salaire minimum au Sénégal. Il s'avère que cette orientation presque exclusivement commerciale permet indiscutablement aux ménages agri-urbains de mieux couvrir leurs dépenses alimentaires et non-alimentaires inhérentes à la vie en milieu urbain.
\end{abstract}

Mots clefs : agriculture urbaine et périurbaine, économie des ménages agri-urbains, Dakar, Sénégal.

\section{Urban and peri-urban agriculture (AUP) and the economy of agri-urban households in Dakar (Senegal)}

\begin{abstract}
The current dynamics of urbanization in sub-Saharan Africa pose significant challenges to food and food security for urban populations. In this context, the reflection on the food function of urban and peri-urban agriculture (UPA) is highly relevant and much work has been devoted to analysing the food function of the UAP. On the other hand, little research has been carried out on the essentially commercial dimension of the activity, making it possible to generate income likely to meet the different needs, food and non-food, of agri-urban households. This contribution focuses on this particular aspect of market gardening activity carried out in the Dakar region in three production areas involving 214 market gardeners.

The results obtained show that the UPA through urban market gardening in Dakar constitutes a fully-fledged commercial activity whose monetary income is far higher than the minimum wage in Senegal. It turns out that this almost exclusively commercial orientation undoubtedly allows agri-urban households to cover their food and non-food expenses inherent in living in urban areas.
\end{abstract}

Keywords-Urban an peri-urban agriculture, agri-urban households economy, Dakar, Senegal.

\section{INTRODUCTION}

Avec plus de 7 milliards d'habitants (UNFPA, 2016), la planète est aujourd'hui confrontée à un problème de satisfaction des besoins alimentaires de sa population (Bricas et Seck, 2004). Le problème de la sécurité alimentaire mondiale se pose de plus en plus avec acuité au niveau des villes devenues en l'espace de quelques années le lieu de vie de plus de la moitié de la population (Veron, 2007). La sécurité alimentaire en milieu urbain est encore plus préoccupante dans les villes des pays du Sud, déjà confrontées à un taux de pauvreté important. C'est dans ce contexte que l'agriculture urbaine et périurbaine (AUP) se développe en occupant une place incontournable dans l'approvisionnement alimentaire des villes (Mougeot, 
2000, Aubry et al., 2010). L'AUP que nous définirons ici comme l'ensemble des activités agricoles situées à l'intérieur ou en périphérie de la ville (Mbaye et Moustier, 2000) représenterait entre le tiers et le quart de la consommation mondiale de produits agricoles (Padilla, 2005).

La fonction alimentaire de l'AUP a été largement étudiée dans plusieurs recherches le plus souvent consacrées au contexte des villes situées dans les pays du Sud (Moustier et Pages, 1997, Aubry et al., 2010, De Bon et al., 2010, Chagomoka et al., 2015). Dans la plupart des travaux, cette fonction de l'AUP est abordée sous l'angle du rôle qu'elle joue par rapport à la sécurité alimentaire des populations urbaines, en particulier dans les stratégies des ménages urbains pauvres (Casale, 2006, Mfoukou-Ntsakala A. et al., 2006, Olahan, 2010, Chagomoka et al., 2015). Pour autant, dans un contexte urbain marqué par l'omniprésence des échanges commerciaux et monétaires, limiter la fonction alimentaire de l'AUP à son caractère d'activité d'autoconsommation paraît réducteur. Elle se décline également en une activité génératrice de revenus (Maxwell, 2003) permettant aux ménages agricoles urbains de diversifier considérablement leurs régimes alimentaires (Zezza \& Tasciotti, 2010). Dans ce cadre, la forme marchande et commerciale de l'AUP est un atout majeur pour les ménages agricoles urbains. Or, bien que beaucoup évoquée (Golhor, 1995, Temple et Moustier, 2004, Smith, 2004, Ba et Aubry 2011), cette question reste peu abordée dans la littérature (Danso et al., 2002, Zezza et Tasciotti, 2010, Dubbeling et al., 2010).

À Dakar, l'agriculture, particulièrement le maraîchage, est une pratique très ancienne et bien ancrée dans l'écosystème urbain et périurbain. D'abord développée du temps de la colonisation (Sposito, 2010), elle a été vite adoptée par les populations locales particulièrement celles issues de l'exode rural et jadis agriculteurs dans leurs villages. Pour autant, le maraichage urbain dans la capitale sénégalaise présente d'importantes particularités si on la compare avec des activités agricoles urbaines menées dans d'autres villes. Que ce soit à Antanarivo, Ouagadougou ou Brazzaville (Madjélia et al., 2016, Aubry et al., 2008, Mfoukou-Ntsakala et al., 2006) l'AUP y est menée et perçue comme une activité d'autoconsommation garantissant aux ménages urbains les plus pauvres une sécurité alimentaire grâce à un accès direct à des aliments frais. Or, l'horticulture maraîchère à Dakar sort de ce stéréotype. Même si elle occupe une place prépondérante en termes d'approvisionnement alimentaire en produits frais et de création d'emplois pour la ville (Temple et Moustier, 2004, Guèye et al, 2009, IAGU, 2011), elle constitue une activité essentiellement commerciale. La faiblesse de la part autoconsommée des productions maraichères s'explique en partie par les habitudes alimentaires observées dans la région de Dakar (Sposito, 2010, GRDR, 2015). A titre de comparaison, si les légumes cultivés dans ce territoire sont consommés par les populations locales, elles ne sont pas par contre des aliments de base dans les repas dakarois contrairement au riz et au cresson cultivé, dans la ville d'Antanarivo (Dabat et al., 2012, Aubry et al., 2008). Le principal aliment de base à Dakar et de manière générale au Sénégal est le riz majoritairement importé des pays asiatiques même si la production locale provenant de la vallée connait de plus en plus de succès. Et cette importance des produits importés ne se limite pas au riz car la part des produits alimentaires dans les importations du Sénégal est d'environ 54\% (Wade \& Lançon, 2015). Malgré le caractère commercial de l'AUP, l'analyse des revenus issus des activités de maraîchage n'a été abordée que dans deux études relativement récentes (IAGU, 2011 ; Gaye et Niang, 2010). Par contre, la question spécifique de l'impact de ces revenus sur l'économie des ménages et ses implications en termes de sécurité alimentaire n'a pas été traitée. Le présent article ambitionne de mieux documenter la pratique de l'AUP dans la région de Dakar et d'éclairer sa contribution économique à la satisfaction des besoins des ménages agri-urbains. Une première partie détaillera les principales caractéristiques de l'AUP dans la région dakaroise. Une deuxième partie présentera un bilan socioéconomique et discutera l'hypothèse d'une vocation essentiellement commerciale de cette activité, dont les recettes permettent aux ménages agri-urbains de mieux répondre à leurs différents besoins non-alimentaires, tout en contribuant au renforcement de la sécurité alimentaire. Une dernière partie traitera finalement des contraintes qui pèsent sur le devenir de l'AU dans la région de Dakar.

\section{MATÉRIAUX ET MÉTHODES}

\section{Unité de sondage et taille de l'échantillon}

L'importance des zones de production maraîchère dans la région de Dakar $(\mathrm{Ba}, 2008)$ fait que le choix de l'unité de sondage sur le terrain devient crucial pour la fiabilité des résultats. L'étude s'est fondée sur une discrimination spatiale des sites de production en suivant la subdivision administrative de la région de Dakar. Celle-ci a permis de classer les futurs sites sélectionnés selon un gradient urbain/rural. Vu l'existence de plusieurs sites de production dans chaque niveau, le critère d'importance ou d'envergure du site de production en termes de superficie occupée a été introduit. Le croisement de ces critères a permis de ressortir trois unités de sondage que nous qualifierons indifféremment tout au long de cet article sous le vocable de sites ou zones de production. Ces zones de production sont respectivement la Grande Niayes de Pikine appartenant à la partie intra-urbaine de Dakar, les Niayes de Malika située en zone péri-urbaine et la zone de 
Lendeng de Rufisque à une quarantaine de kilomètres dans la partie rurale de la région.

L'échantillon, défini suivant une méthode statistique usuelle $^{1}$, se compose de 214 individus, répartis comme suit : 101 maraîchers dans la Grande Niayes de Pikine, 54 à Malika et 59 à Lendeng.

\section{Déroulement des enquêtes}

Les données ont été recueillies au moyen de questionnaires et d'entretiens semi-directifs avec 12 focus groupes, entre mars et juin 2016. L'élaboration du questionnaire, la saisie et l'apurement de la base de données a été faite sur Sphinx. L'ensemble des traitements statistiques ont été mené sur Excel et SPSS.

\section{Présentation du cadre de l'étude}

La région de Dakar couvre une superficie de $550 \mathrm{~km}^{2}$, soit $0,3 \%$ du territoire national et sa population est estimée en 2017 par l'ANSD à 3529300 habitants soit $49 \%$ de la population urbaine du Sénégal. Elle concentre également l'essentiel des services administratifs, du tissu industriel et des établissements commerciaux ainsi que financiers du pays. Cette situation ne fait que renforcer la forte croissance urbaine qu'on enregistre aujourd'hui dans la région de Dakar avec un niveau d'urbanisation de 96\%. Malgré ce caractère de région quasiment urbaine, la pratique agricole, notamment la culture maraîchère, constitue une activité majeure dans l'économie de la région, marquée par un secteur informel prédominant et un taux de chômage de la population assez élevé (15,7\%) surtout au niveau des personnes de moins de 30 ans (ANSD, 2017). Ainsi, on dénombre à l'échelle de la région de Dakar plus d'une vingtaine de sites ou zones de production agricoles $(\mathrm{Ba}, 2008)$ situées pour l'essentiel dans le domaine agroécologique des Niayes.

\section{La Grande Niayes de Pikine}

Située dans la partie ouest de Dakar, la Grande Niayes de Pikine est la plus grande zone des Niayes de cette région et couvre une superficie de 750 ha. Elle est limitée au nord par la commune de Golf Nord (département de Guédiawaye), au Sud par la Patte d'Oie (département de Dakar), à l'est par la ville de Pikine et à l'ouest par le village de Cambérène (département de Dakar). À l'image de l'ensemble des Niayes, la Grande Niayes de Pikine alterne un ensemble de systèmes dunaires et de dépressions interdunaires. Si les dunes sont composées de sols à faible capacité de rétention d'eau, celui des cuvettes et marigots interdunaires est essentiellement argileux ce qui explique son fort potentiel agricole. Les Niayes se distinguent également par le caractère affleurant de la nappe phréatique qui permet aux maraichers de s'adonner quasiment toute l'année à leur activité agricole. La Grande Niayes de Pikine est l'une des plus grandes zones de production maraîchère de la région de Dakar et la plus grande située en milieu intra-urbain (Faye, 2010).

\section{La zone de Malika}

La zone de production de Malika se trouve dans la commune du même nom située à environ une vingtaine de kilomètres du centre-ville de Dakar dans la périphérie du département de Pikine. Elle fait également partie de l'ensemble des Niayes et connait donc les mêmes problématiques associées à cet ensemble. Malika abrite également la principale décharge d'ordures sauvages de la région de Dakar, Mbeubeuss, sur le lit d'un ancien lac autour duquel se développent les activités agricoles. Par ailleurs, Malika est confronté aux effets du changement climatique avec l'avancée de la mer qui, cumulée au caractère affleurant de la nappe phréatique, font que les ressources hydriques de cette zone sont très vulnérables aux phénomènes de salinisation (Ba et al., 2016), observé sur l'ensemble des Niayes.

\section{La zone de Lendeng}

La zone de Lendeng se trouve dans la commune de Rufisque Est dans le département de Rufisque à environ une quarantaine de kilomètres de Dakar. Elle appartient au même écosystème des Niayes, ce qui lui confère des conditions physiques adéquates pour la pratique agricole notamment la production maraîchère. Contrairement aux autres zones des Niayes, la nappe phréatique y est plus profonde car allant de 12 à 36 mètres, ce qui ne facilite pas son accès par les céanes ${ }^{2}$ et/ou les puits pour une exploitation agricole. Par ailleurs, la zone agricole de Lendeng s'étend sur une superficie de 56,83 ha dont 40 ha exploitées à des fins de production maraîchères soit $70 \%$ de l'espace disponible (IAGU, 2011). Lendeng est également la plus importante zone de production maraîchère de la région de Dakar en termes de disponibilités foncières et en termes de quantités produites.

${ }^{1}$ Méthode de Bernoulli

${ }^{2}$ Terme désignant un puits peu profond creusé dans le sol avec une forme plus ou moins évasée suivant sa profondeur. 


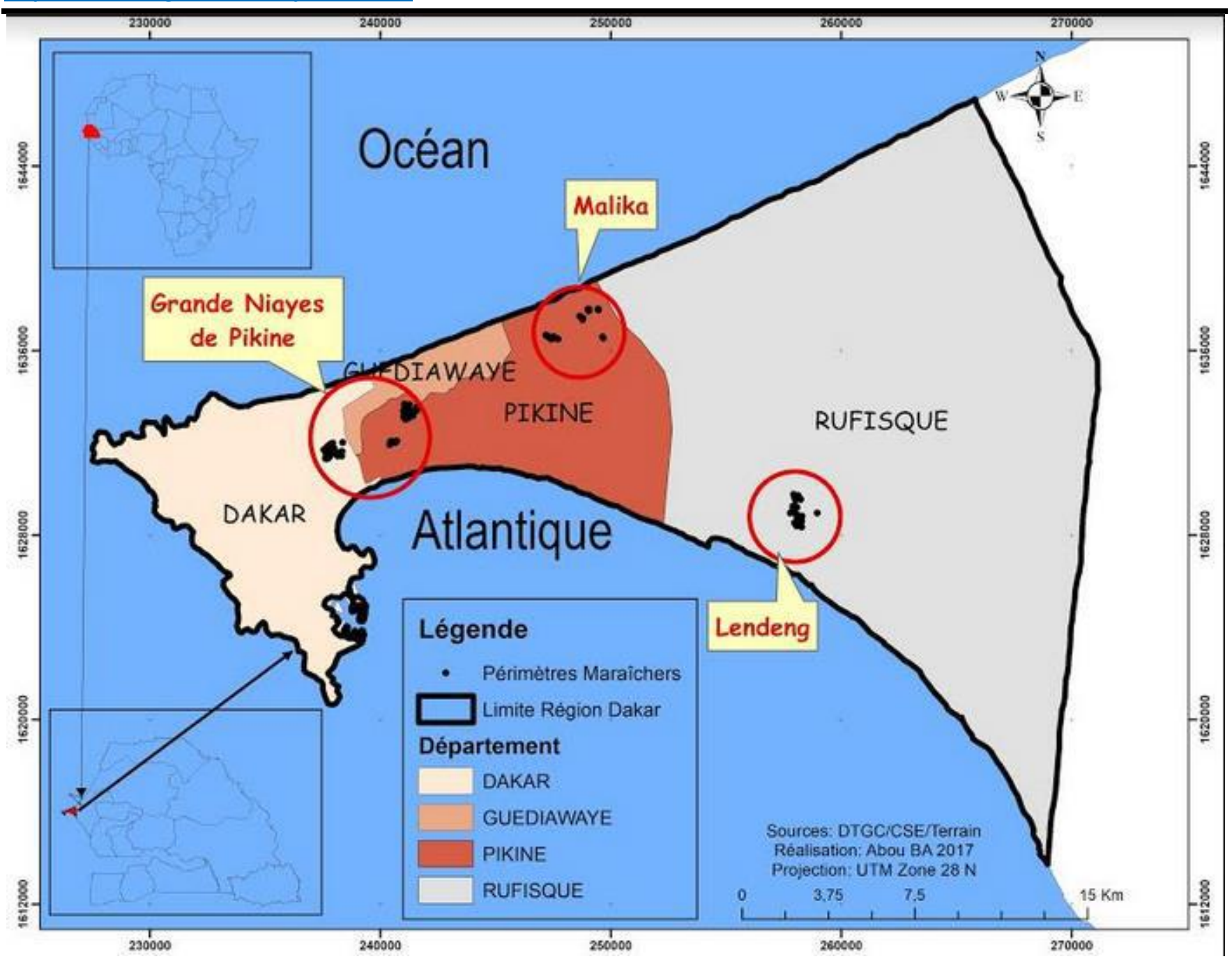

Fig.1: Carte de situation de la région de Dakar et de localisation des zones d'étude

Caractéristiques de l'agriculture urbaine et périurbaine à Dakar

\section{Une activité masculine peu attractive pour les jeunes}

L'AUP à Dakar particulièrement l'horticulture maraîchère est une activité presque exclusivement (à 95\%) masculine. Cela s'explique par le contexte socioculturel du Sénégal où, d'une part, les femmes ont difficilement accès à la terre agricole et, d'autre part, elles sont généralement spécialisées dans la commercialisation des récoltes (Niang, 2014). Ces agriculteurs sont généralement des adultes avec un âge moyen de 49 ans (âge minimum de 19 ans et maximum de 80 ans). Au-delà de cet âge moyen, on constate que malgré l'importance du chômage chez les jeunes, le maraîchage ne mobilise que très peu cette catégorie de la population (18\% des agriculteurs interrogés). Cette situation résulte d'abord de la précarité de cette activité ensuite de sa marginalisation par les pouvoirs publics (Kedowide, 2010) et enfin de l'omniprésence des séniors, voire des retraités, demandeurs de revenus supplémentaires (Bâ et al, 2016) dans un contexte où le système de retraite est très largement inexistant.

Des acteurs expérimentés et animés par une question de survie

Le maraîchage à Dakar est une activité menée principalement par des agriculteurs très expérimentés. Près de la moitié des maraichers interrogés (46\%) ont une vingtaine d'années révolues dans la pratique agricole en milieu urbain alors que seulement près de $8 \%$ (tableau 1 ) y sont depuis moins de cinq années. Ceci dénote entre autres du manque de renouvellement des acteurs dans cette filière consécutive probablement à la réduction des espaces agricoles née de l'urbanisation ininterrompue de l'agglomération dakaroise.

Tableau 1 : Nombre d'années passées dans l'activité agricole

\begin{tabular}{|c|c|c|c|c|c|c|}
\hline Catégorie & {$[\mathbf{0 - 5}]$} & {$[\mathbf{6 - 1 0}]$} & {$[\mathbf{1 1 - 1 5}]$} & {$[\mathbf{1 6 - 2 0}]$} & [21 et plus] & Total \\
\hline Effectifs & 16 & 42 & 26 & 31 & 99 & 214 \\
\hline Proportion (\%) & 7,5 & 19,6 & 12,1 & 14,5 & 46,3 & 100 \\
\hline
\end{tabular}


Les considérations liées à la survie sont les principales motivations des agriculteurs dakarois à s'adonner à l'horticulture maraîchère. En effet, le besoin de subsistance est le motif le plus cité avec $81 \%$ des réponses enregistrées. Cela est davantage conforté par plusieurs recherches qui voient en l'AUP une activité de subsistance pour les ménages urbains les plus démunis (Olahan, 2010, Chagomoka et al, 2015), même si le contexte dakarois montre plusieurs spécificités.

\section{Une situation foncière précaire et ambiguë}

La situation foncière permet d'interroger les modalités d'accès à la terre des agriculteurs urbains de la région de
Dakar. Les résultats montrent une typologie du statut foncier très hétérogène entre les agriculteurs. C'est ainsi que la plupart des agriculteurs (51\%) se considèrent comme des propriétaires de leur exploitation contre, $15 \%$ de métayers, $11 \%$ de locataires et $6 \%$ d'agriculteurs se considérant être dans une situation d'occupation anarchique (figure 2). Il convient de noter que sur les $51 \%$ d'agriculteurs se disant propriétaires, moins de $20 \%$ disposent d'actes de propriété. Et parmi ces derniers, une majorité s'avère contestable d'un point de vue juridique, le transfert de propriété reposant généralement sur des actes non reconnus par la législation foncière.

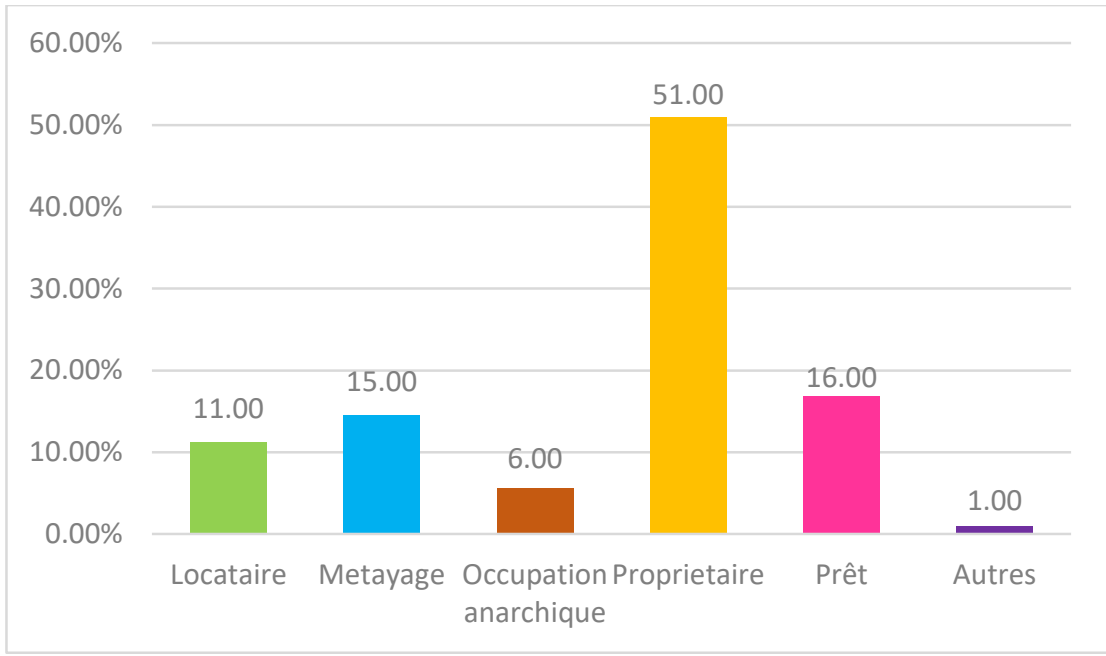

Fig.2 : Répartition des agriculteurs selon leur statut foncier

Par ailleurs, le caractère informel et/ou traditionnel des formes de tenure foncière accentue la précarité de la situation des agriculteurs puisqu'elle ne leur permet pas d'obtenir des crédits au niveau des institutions financières en mettant sous hypothèque ou sous garantie leur exploitation agricole. De plus, l'exiguité des superficies agricoles exploitées constitue également un frein pour les bailleurs qui préfèrent investir dans les grandes exploitations agricoles. Les activités maraîchères se font en effet sur de petites exploitations avec en moyenne 0,39 ha par agriculteur. La distribution des agriculteurs selon la superficie exploitée est assez hétérogène (écart-type $=0,54$ ha) même si près de six (06) agriculteurs sur dix (10) exploitent moins de 0,25 ha (tableau 4 ).

Tableau 2 : Répartition des agriculteurs selon la superficie cultivée

\begin{tabular}{|l|c|c|}
\hline Superficie cultivée (ha) & Effectifs de producteurs & Proportion (\%) \\
\hline$[0-0,25]$ & 124 & 57,9 \\
\hline$] 0,25-0,5]$ & 28 & 13,1 \\
\hline$] 0,5-0,75]$ & 27 & 12,6 \\
\hline$] 0,75-1,0]$ & 5 & 2,3 \\
\hline$] 1,0-1,25]$ & 15 & 7,0 \\
\hline$] 1,25$ et plus $]$ & 15 & 7,0 \\
\hline Total & 214 & 100 \\
\hline
\end{tabular}

\section{Les sources et modes d'irrigation}

On recense trois sources d'approvisionnement en eau dans l'AUP à Dakar. La plus répandue est basée sur l'utilisation de la nappe phréatique (Bâ et al., 2016), peu profonde dans la région du fait de son appartenance à la grande zone 
agroécologique des Niayes. La deuxième source est le réseau de distribution d'eau potable assurée par la $\mathrm{SDE}^{3}$. Celle-ci est beaucoup plus présente dans la partie sud des Niayes à cause de la salinité des sols et des eaux souterraines. Les agriculteurs ayant recours à cette source d'approvisionnement sont soumis à d'énormes contraintes à cause de la concurrence avec les besoins domestiques, même s'ils bénéficient d'un système tarifaire préférentiel contrôlé. La dernière source d'approvisionnement en eau à Dakar est relativement nouvelle et très peu répandue à l'échelle de la région. Il s'agit de l'utilisation des eaux usées traitées (EUT) fournies depuis 2012 par les STEP ${ }^{4}$ grâce à un programme initié par la FAO ayant permis l'installation d'un réseau d'adduction allant des STEP aux zones de production concernées (Souré et al., 2012).

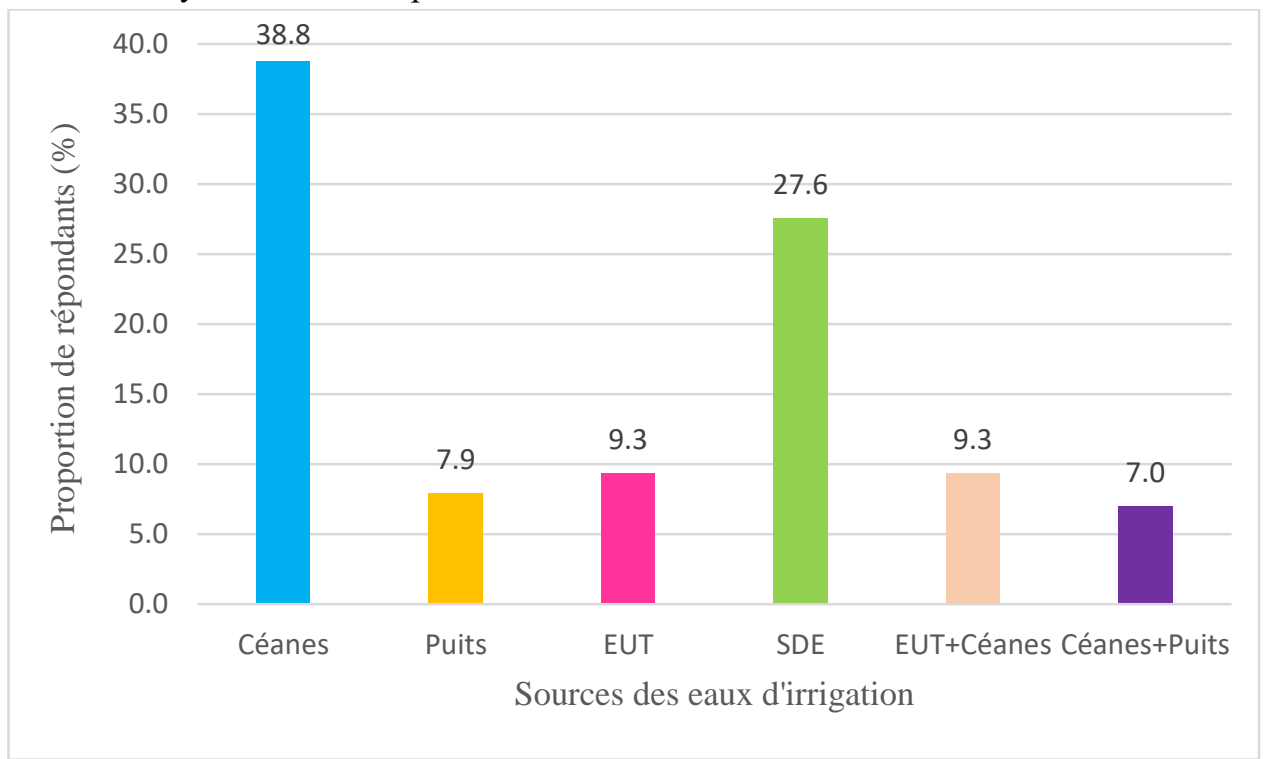

Fig.3: Principales sources d'irrigation

Les déchets organiques urbains comme matières premières pour la fertilisation

L'utilisation des déchets organiques dans le maraichage n'est pas une pratique nouvelle, mais elle occupe de plus en plus de place dans les activités maraîchères et en dehors, car participant à l'amélioration du cadre de vie urbain par le recyclage des milliers de tonnes de déchets produits chaque jour dans la ville (Tounkara, 2015, N’Diénor, 2014). Ces fertilisants sont très divers de par leur origine, mais ce sont les crottins de cheval qui en représentent la plus grande proportion avec $71 \%$, suivis des fientes de volaille avec $54 \%$ et des bouses de vache utilisées par 22 $\%$ des agriculteurs. Cette importance des fertilisants organiques (figure 4) s'explique d'une part par leur disponibilité et d'autre part par la forte intégration de l'horticulture maraîchère au secteur de l'élevage.

Malgré, la pratique répandue de la fertilisation organique, on note toujours la persistance de la fertilisation chimique ou minérale. Cette dernière concerne principalement deux fertilisants : l'urée et le NPK ${ }^{5}$, utilisés par l'ensemble des agriculteurs interrogés. Ce constat est similaire à celui fait dans les travaux de Niang (2014) et du IAGU (2011).

\footnotetext{
${ }^{3}$ Sénégalaise des eaux, société publique de distribution d'eau potable
}

${ }^{5}$ Engrais composé de trois éléments chimiques : azote $(\mathrm{N})$, phosphore $(\mathrm{P})$ et potassium $(\mathrm{K})$ 


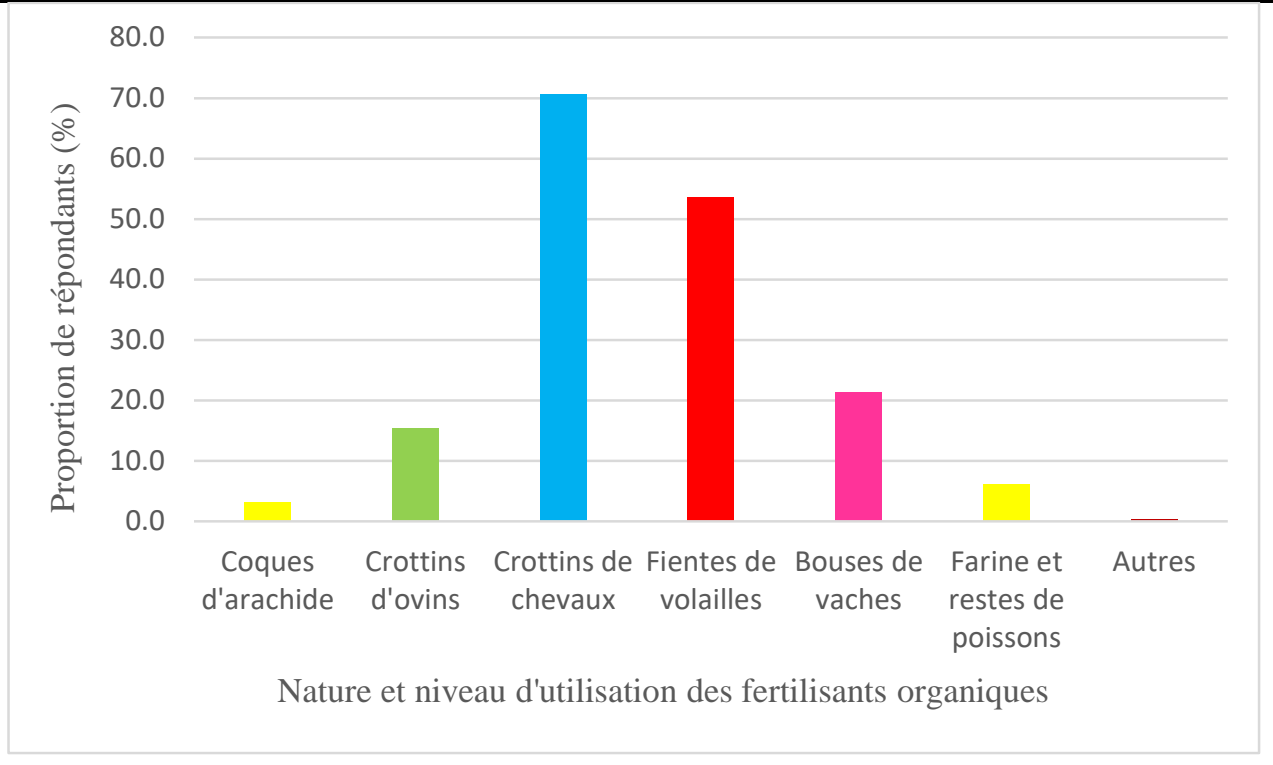

Fig.4: Nature et niveau d'utilisation des fertilisations organiques

\section{Aperçu des principales spéculations cultivées}

Du fait de son caractère d'activité urbaine, l'horticulture maraîchère à Dakar est dominée par des spéculations à cycle relativement court. Ces dernières peuvent être regroupées en deux catégories : les légumes à feuilles (les plus cultivées) et les légumes à fruit. Pour les premières, la laitue, le chou et la menthe constituent les principales spéculations avec respectivement $88 \%, 34 \%$ et $22 \%$ de part d'activité chez les agriculteurs. Tandis que pour les légumes à fruit, ce sont l'oignon (54\%), le jaxatou ${ }^{6}(48 \%)$ et le poivron (45\%) qui occupent plus les maraîchers (figure 5).
On constate que la localisation est un élément important dans le choix des spéculations cultivées à cause entre autres de la pression foncière, du besoin de répondre à la forte demande des citadins et de la nécessité d'avoir des revenus en permanence pour financer les activités agricoles. C'est ainsi que dans les zones intra-urbaines (Grande Niayes de Pikine), on a une omniprésence des légumes feuilles à cycle très courts, particulièrement la laitue et la menthe. Par contre, les zones périurbaines présentent une plus grande variété de spéculations avec des cycles de cultures plus longs même si la laitue y occupe à l'instar de toute la région une place de choix dans le calendrier des agriculteurs.

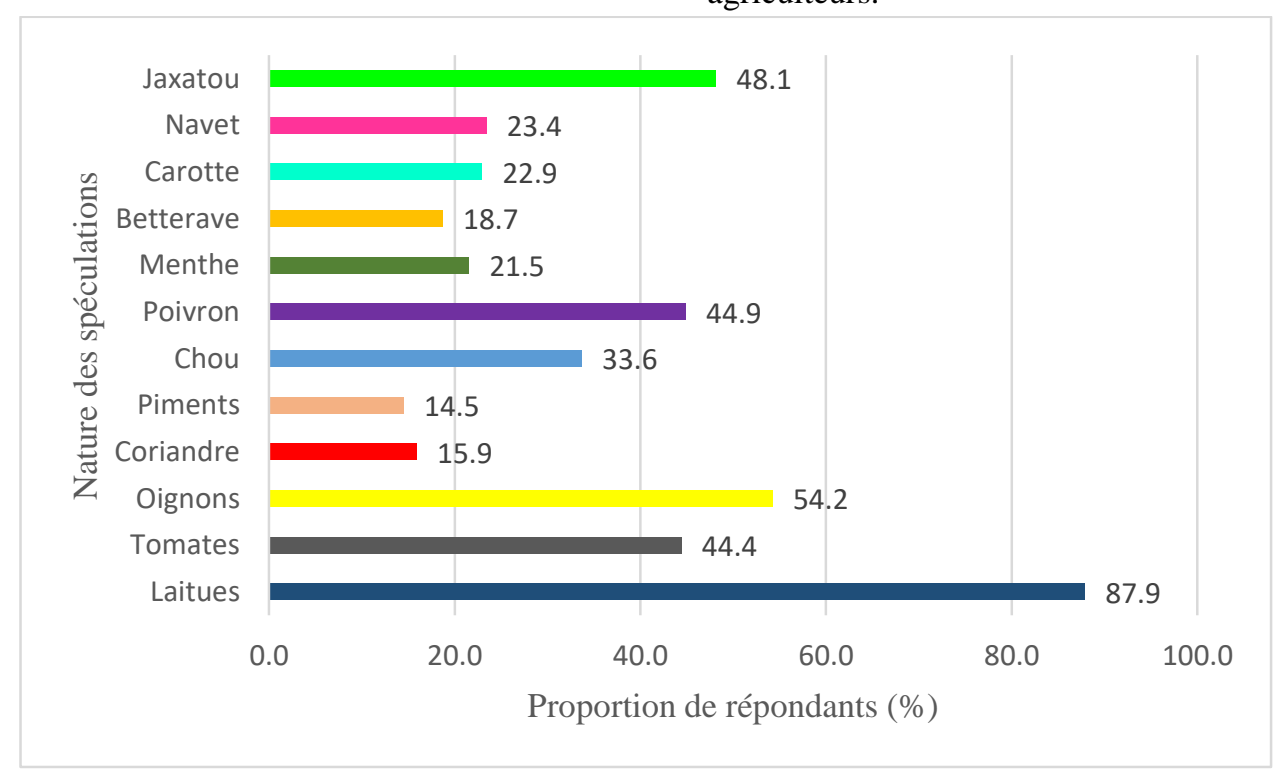

Fig.5: Type de spéculations cultivées

Principales contraintes rencontrées par l'AUP

${ }^{6}$ Aubergine amère 
L'horticulture maraichère, à l'image de toutes les activités agricoles pratiquées dans la région de Dakar, doit faire face à d'importantes contraintes dont la plupart sont inhérentes à son caractère d'activité urbaine. Les trois principales contraintes identifiées (figure 6) par les agriculteurs sont l'insécurité foncière $(74 \%)$, le manque d'eau pour l'irrigation (62\%) et la salinisation des eaux et des sols (27 $\%)$. Ces résultats corroborent les travaux antérieurs de Gaye et Niang (2010), IAGU (2011) et Niang (2014). D'autres contraintes moins préoccupantes que les trois principales entravent également le bon fonctionnement des activités maraichères. Il s'agit entre autres de l'accès aux systèmes de crédits (19\%), des contraintes climatiques principalement les inondations (18\%) et de la limitation des quotas pour les exploitations faisant l'irrigation avec l'eau de la SDE (15\%). À cela, on peut ajouter d'autres contraintes propres à chaque zone de production comme la cherté de l'eau pour les agriculteurs utilisant l'eau de la SDE et la mauvaise qualité des eaux usées traitées pour les maraîchers de la grande Niayes de Pikine.

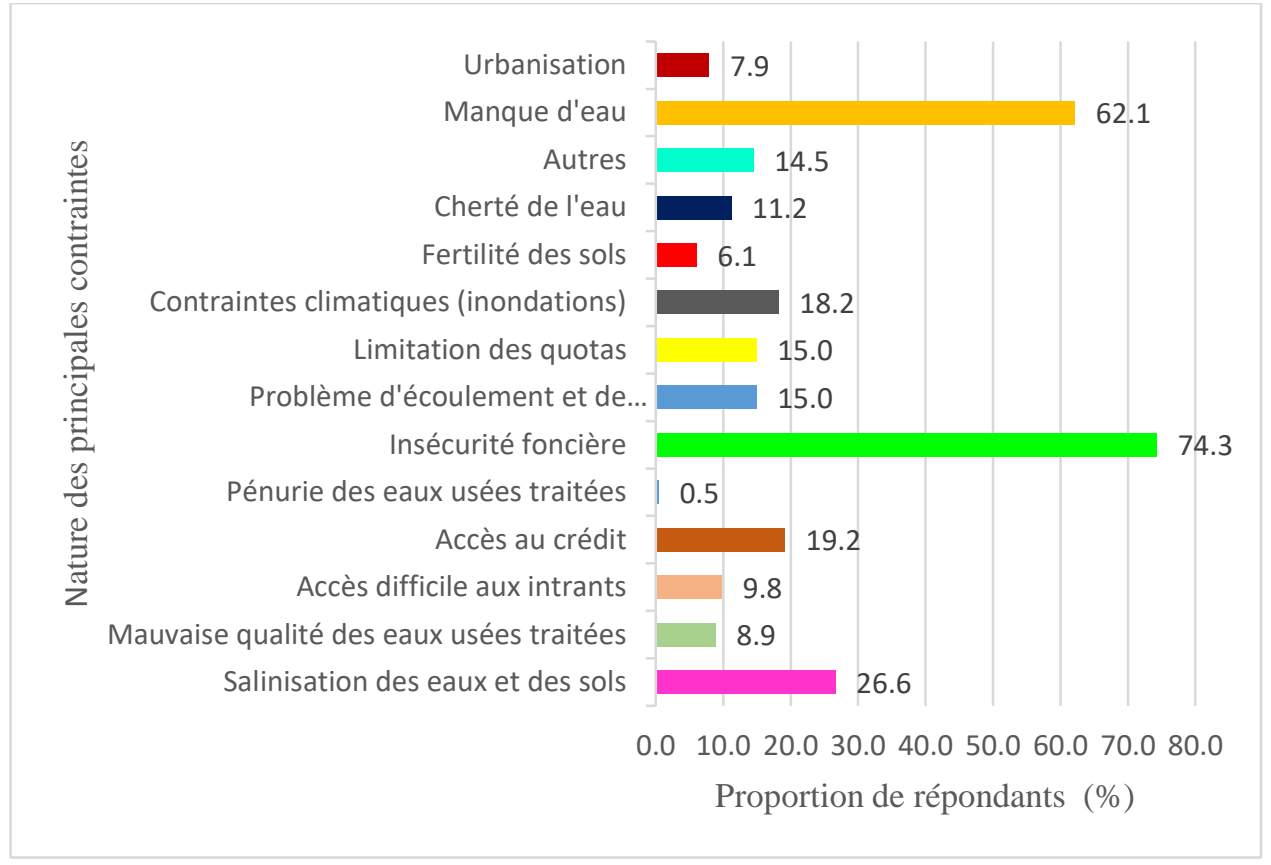

Fig.6: Nature et niveau d'importance des contraintes pour les agriculteurs

\section{Les fonctions reconnues aux zones de production agricoles à Dakar}

L'AUP est une activité remplissant diverses fonctions au niveau des territoires dans lesquels elle se développe. Cette multifonctionnalité, bien relatée dans la recherche (Duchemin, 2013, Ba, 2011, Duchemin \& al, 2008) renseigne par contre très peu sur les perceptions ou les fonctions que les agriculteurs assignent à leurs zones de production. Or, il apparaît que les agriculteurs dakarois considèrent principalement les espaces qu'ils occupent en milieu ou en périphérie de la ville comme des zones de production agricoles à part entière (figure 7). Tandis que $70 \%$ d'entre-eux mettent en avant la fonction de zone génératrice de revenus, l'importance de la fonction environnementale dans les choix des agriculteurs est également soulignée. En effet, 45 et $44 \%$ des agriculteurs voient leur espace agricole respectivement comme une zone de recyclage des déchets urbains et un espace vert pour la ville. La fonction éducative ressort également dans les résultats (25\% des agriculteurs) comme support pédagogique pour les élèves et étudiants de la région. Seuls $18 \%$ des agriculteurs voient leur espace agricole comme une réserve foncière de la ville, qui sera appelée tout ou tard à disparaître au profit de projets immobiliers ou d'infrastructures majeures. En comparant ces résultats avec les travaux menés dans quelques zones agricoles de la région de Dakar (Ba, 2011 ; IAGU, 2011), on constate un profil tout à fait similaire en termes de réponses. 


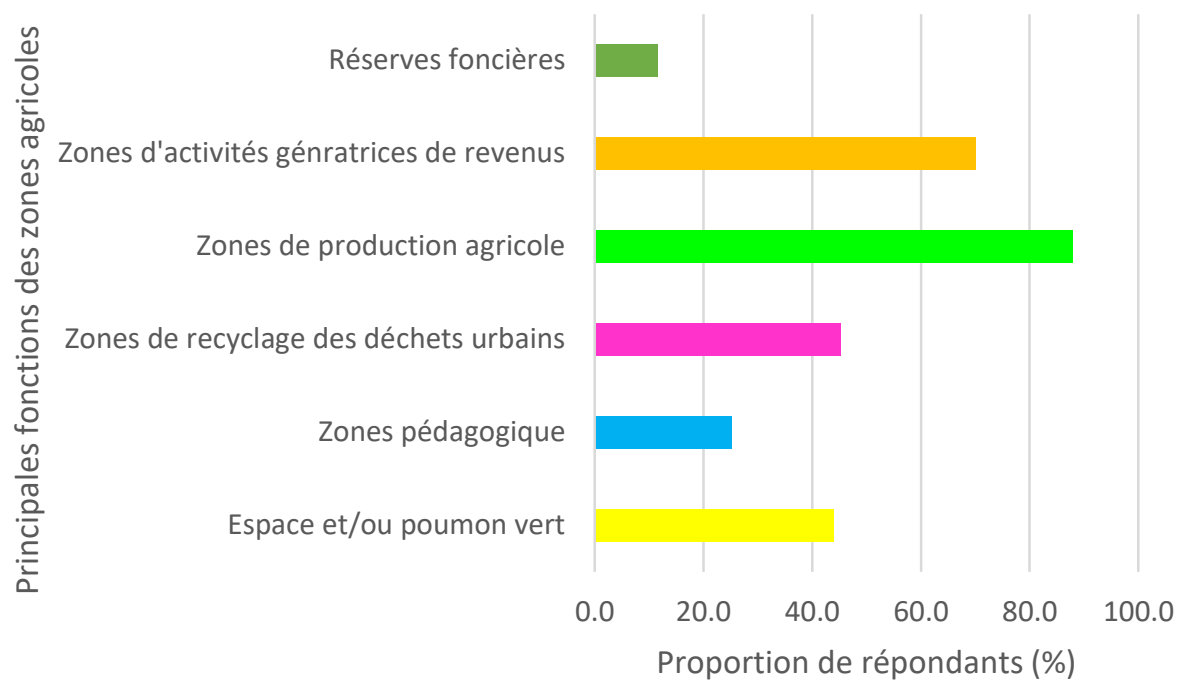

Fig.7: Principales fonctions reconnues aux zones agricoles par les producteurs

\section{Bilan socioéconomique du maraîchage urbain}

L'évaluation économique du maraîchage urbain à Dakar a été menée à travers des enquêtes quantitatives rétrospectives menées entre les mois de mars et juin 2016. Les résultats concernent donc la campagne agricole précédente à la période de déroulement de l'enquête. Cette méthode d'évaluation économique présente des inconvénients car elle n'est basée que sur les propos recueillis auprès des producteurs dans un contexte où la question des revenus est généralement taboue. Toutefois, elle a l'avantage de permettre de comprendre au mieux les performances économiques de cette activité, pratiquée très souvent par des acteurs qui ne tiennent aucun compte d'exploitation. La non-tenue d'un compte d'exploitation par les producteurs et la volatilité des prix des légumes d'une saison à l'autre, voire même au cours d'une semaine, rendent difficile tout calcul d'un résultat net de production.
Pour pallier cela, l'étude s'est basée sur une estimation par les producteurs de leurs coûts de production et de leurs bénéfices nets par campagne. Les coûts de production sont l'ensemble des charges qui entrent directement dans le fonctionnement de l'exploitation maraîchère tandis que le bénéfice constitue le revenu gagné par le producteur après déduction de ces charges de production. Par ailleurs, la diversité des exploitations agricoles des zones étudiées explique le choix de présenter le profil économique du maraichage urbain à Dakar par zone de production. Cette diversité s'exprime également en termes de capacités d'investissement et de production. C'est ainsi que la zone de Lendeng bénéficiant des plus importantes superficies agricoles par rapport à celles de Malika ou de la grande Niayes de Pikine, enregistre les charges et les résultats nets de production les plus importants (tableau 3$)^{7}$. Cela traduit également de plus grandes disponibilités financières pour réaliser les investissements requis.

Tableau 3 : Compte d'exploitation d'un maraîcher moyen par campagne (en euros)

\begin{tabular}{|l|r|r|r|r|}
\hline Rubriques & \multicolumn{1}{l|}{ Malika } & \multicolumn{1}{l|}{ Niayes de Pikine } & \multicolumn{1}{l|}{$\begin{array}{l}\text { Mufisque (Lendeng) } \\
\text { trois zones }\end{array}$} \\
\hline Semences & 64,80 & 109,70 & 208,40 & 125,30 \\
\hline Engrais et phyto & 22.76 & 37,40 & 76,20 & 44,40 \\
\hline Fumure organique & 54,11 & 82,30 & 106,30 & 95,60 \\
\hline Charges d'irrigation & 12,43 & 26,40 & 274,90 & 91,40 \\
\hline Salaires personnel & 43,93 & 73,30 & 199,90 & 103,00 \\
\hline Préparation du sol & 3,75 & 12,30 & 71,30 & 28,30 \\
\hline Total des charges & 201,78 & 348,80 & 986,90 & 487,90 \\
\hline Résultat net/campagne & 764,50 & 975,50 & 2136,10 & $\mathbf{1 ~ 2 4 2 , 2 0}$ \\
\hline Revenu net annuel & 3076,80 & 4044,00 & 8544,40 & $\mathbf{4 9 6 8 , 8 0}$ \\
\hline
\end{tabular}

\footnotetext{
${ }^{7}$ Les valeurs sont en euros et 1 euro $=655$ FCFA
} 
Des revenus agricoles au-dessus de la moyenne dakaroise

L'esquisse du compte d'exploitation des maraichers montre que le revenu net moyen par campagne obtenu à l'échelle des trois zones d'étude est de 1242 euros. Sachant que près de $98 \%$ des producteurs interrogés mènent quatre campagnes par année, on obtient un revenu net annuel moyen pour un maraîcher de 4969 euros soit un revenu mensuel de 414 euros. En comparaison, le salaire minimum interprofessionnel garanti (SMIG) dans le pays et le salaire moyen d'un employé sont respectivement de 55 et 174 euros (ANSD, 2017) donc largement inférieurs au revenu net moyen d'un maraîcher. Ce revenu constitue aussi l'essentiel des ressources des ménages agri-urbains. Il représente en effet $82.5 \%$ des dépenses de consommation, la partie restante étant assurée par des revenus issus d'autres activités, qu'environ $50 \%$ des ménages agrourbains déclarent pratiquer. Les disponibilités financières des ménages agri-urbains s'avèrent ainsi être très proches de celles des ménages de la région dakaroise dans son ensemble. Si l'on compare les dépenses de consommation des ménages (nous ne disposons pas de données sur les revenus des ménages dans la région de Dakar) le différentiel est minime, de l'ordre de $2 \%$ (tableau 4 ciaprès) ${ }^{8}$.

Tableau 4 : Structure des principaux postes de dépense des ménages agri-urbains et urbains de Dakar

\begin{tabular}{|l|r|r|r|r|}
\hline \multirow{2}{*}{ Types de dépenses } & \multicolumn{2}{|l|}{$\begin{array}{l}\text { Ensemble ménages } \\
\text { de Dakar }\end{array}$} \\
\cline { 2 - 5 } & Montants (en euros) & Part (\%) & Montants (en euros) & Part (\%) \\
\hline Alimentation & 2677,20 & 44,4 & 2290,40 & 38,9 \\
\hline Éducation & 324,20 & 5,4 & 270,80 & 4,6 \\
\hline Santé & 156,30 & 2,6 & 194,30 & 3,3 \\
\hline Transport & 234,60 & 3,9 & 370,90 & 6,3 \\
\hline Communication & 78,30 & 1,3 & 29,40 & 0,5 \\
\hline $\begin{array}{l}\text { Logement (électricité+eau } \\
\text { +autres combustibles, } \\
\text { meubles+articles de ménage) }\end{array}$ & 2248,50 & 37,3 & 2019,60 & 34,3 \\
\hline Autres dépenses & 307,40 & 5,1 & & 12,1 \\
\hline Total & 6026,60 & 100,0 & & 712,40 \\
\hline Revenu net annuel du maraîchage & 4968,80 & $82.5 \%$ & & 100,0 \\
\hline
\end{tabular}

Il se traduit tout de même par une capacité à investir davantage dans des postes de dépenses centraux pour le bien-être du ménage, avec $17 \%$ de dépenses en plus au niveau de l'alimentation, de $19.5 \%$ pour l'éducation et de $11.3 \%$ en plus pour le logement. Les dépenses de santé sont, étonnamment bien inférieures (-24\%).

Finalement, l'importance des revenus issus du marâichage peut également être appréciée en relation aux seuils de pauvreté appliquée dans la région de Dakar. Au plan général, le revenu agricole est 7\% supérieur au seuil de pauvreté monétaire, fixé à 4630 euros annuels (ANSD, 2013). Au plan des dépenses alimentaires, même en considérant une somme légèrement inférieure à celle calculée de notre échantillon, du fait de l'existence de revenus extra-agricoles ${ }^{9}$, le niveau moyen de dépenses

\footnotetext{
${ }^{8}$ Les données régionales sont issues du deuxième rapport de suivi de la pauvreté au Sénégal (ESPS). Le tableau de la structure des dépenses a été modifié dans l'optique de mener une comparaison avec la structure des dépenses issues de nos travaux. Ainsi, certains postes de l'ESPS ont été regroupés à cet effet.
}

serait 13\% supérieur au seuil de pauvreté alimentaire (ANSD, 2013).

Ces différents résultats permettent d'apprécier toute la valeur commerciale du maraîchage urbain et son importance dans l'économie des ménages agri-urbains.

\section{L'AUP : une activité menacée}

Le diagnostic de l'AUP nous a permis d'illustrer toute la dynamique de cette activité, ses impacts sur l'économie des ménages agri-urbains et sa contribution à la sécurité alimentaire des ménages et de la région. Pour autant, l'avenir de ce secteur d'activité est menacé par des contraintes grandissantes, qui, pour partie, dépendent de sa faible reconnaissance par les acteurs en charge de la planification territoriale urbaine.

\section{Des contraintes foncières tenaces}

${ }^{9}$ En l'occurrence, dans la mesure où les revenus agricoles représentent le $82.5 \%$ des dépenses totales, on appliquerait ce même ratio à la ligne budgétaire des dépenses alimentaires. 
La question foncière a toujours été l'un des problèmes majeurs auxquels les activités agricoles menées dans un contexte urbain et/ou périurbain a dû faire face (Diagne 2008 ; Guèye et al., 2009 ; Dauvergne et al., 2010 ; Sposito, 2010). Les contraintes foncières de l'AUP dakaroise sont doubles. En premier lieu elles renvoient à la taille des parcelles. La superficie moyenne ( 0.38 ha par producteur) calculée dans nos enquêtes et qui correspond aux valeurs relevées dans d'autres études (Gaye \& Niang, 2010 ; IAGU, 2011 ; Niang, 2014), est relativement exiguë. A l'heure actuelle, comme démontré, cela est encore suffisant pour couvrir les besoins des ménages agri-urbains. La situation va vraisemblablement changer à l'avenir. D'un côté, les règles en vigueur dans la transmission du capital foncier est susceptible d'amener à son partage parmi les ayants droit. De l'autre côté, la forte dynamique d'urbanisation que connaît la région de Dakar, et qui se traduit par l'arrivée, chaque année, de 100'000 nouveaux habitants (Ba, 2008), induit une demande très importante de surfaces pour la construction de logements et cela se fait systématiquement au détriment des zones agricoles. Cette marginalisation foncière du maraîchage et des producteurs est encore plus accentuée par l'ambiguïté du régime foncier sénégalais (Mendret, 2006 ; Dahou \& Ndiaye, 2009 ; Guèye et al., 2009) et la non-prise en compte de la pratique agricole dans les documents cadre de gestion du territoire (Kedowide et al., 2010). Pour ces différentes raisons, la question de l'insécurité foncière apparaît comme le principal sujet de préoccupation exprimé par les agriculteurs.

\section{Des contraintes d'eau en perpétuelle croissance}

Les contraintes liées à l'eau s'inscrivent dans un contexte de compétition accrue entre les différents usages. L'importante pression démographique qui caractérise la croissance urbaine dakaroise se traduit par une surexploitation de la nappe phréatique dans l'ensemble des Niayes, Dakar y compris, ce qui a conduit à une remontée du biseau salé entrainant un processus de salinisation des réserves d'eau (Guèye-Girardet, 2010) dans les zones jadis très propices au maraîchage. Pour cette raison, les producteurs cherchent des alternatives, en s'équipant en mini-forages permettant de puiser l'eau à une profondeur plus importante (jusqu'à $12 \mathrm{~m}$ ) ou en se raccordant au réseau de distribution de l'eau de la SDE. Cette deuxième solution, malgré son coût plus important, serait de nature à répondre de manière plus adéquate aux besoins en eau des maraîchers urbains. Dans les faits, elle s'avère fragile, tant en raison de la priorité donnée par les instances publiques à la satisfaction des besoins domestiques qu'en raison du déficit dans la distribution d'eau potable (de $162.000 \mathrm{~m}^{3}$ par jour en période de pointe (Sposito, 2010)), dont souffre la région de Dakar. C'est pourquoi, pendant les pics de chaleur de l'été, les zones agricoles comme Lendeng irriguant avec de l'eau fournie par la SDE subissent des coupures récurrentes pouvant durer toute une journée. Pour faire face à cette situation, certains producteurs n'hésitent pas à employer des ouvriers pour s'occuper de l'irrigation pendant la nuit.

\section{CONCLUSION}

Aujourd'hui, les impacts économiques de l'AUP d'une part en termes de productions et d'autre part en termes de revenus financiers particulièrement dans les pays en voie de développement comme le Sénégal ne sont plus à démontrer (Padilla, 2005 ; Aubry et al., 2010 ; De Bon et al., 2010 ; Chagomoka et al., 2015). Plusieurs travaux ont mis en exergue l'apport des activités agricoles urbaines dans l'approvisionnement alimentaire des ménages agriurbains sous le prisme d'une activité d'autoconsommation (Casale, 2006, Mfoukou-Ntsakala et al., 2006, Olahan, 2010). Si cette réalité n'est pas à sous-estimer, force est de constater que l'AUP revêt de plus en plus un caractère marchand dans les pays en voie de développement et récemment dans certains pays développés (Aubry, 2013 ; Toullalan, 2012), ce qui lui permet de mieux jouer son rôle en matière de sécurité alimentaire des ménages agriurbains. Notre recherche démontre que la vocation purement commerciale du maraîchage dakarois, du fait des revenus qu'il génère (cinq à six fois supérieurs au SMIG sénégalais), fait de cette agriculture si particulière une activité urbaine à part entière, à même de faire face à l'ensemble des dépenses des ménages : alimentation, logement, éducation, santé. Et ceci de manière légèrement meilleure que la moyenne des ménages urbains dakarois. A la lumière de ces constats, son maintien, voire son extension, dans le tissu urbain ferait entièrement sens. Paradoxalement, ce qui relève d'une évidence socioéconomique doit de plus en plus composer avec des contraintes environnementales (disponibilité d'eau) et surtout avec une pression foncière constante, qui résulte d'une croissance urbaine chaotique et mal maîtrisée, menaçant, à terme la survie même de l'AUP à Dakar.

\section{REMERCIEMENTS}

Ce travail a bénéficié du soutien financier de la Fondation Ernst et Lucie Schmidheiny que les auteurs tiennent vivement à remercier pour cette contribution. Les auteurs sont grandement redevables à l'ensemble des producteurs maraîchers de Pikine, Malika et Rufisque ainsi qu'à l'ensemble de leurs familles qui ont permis la réalisation de ce travail sur le terrain.

\section{REFERENCES}

[1] ANSD, 2017 : Rapport régional définitif du recensement général de la population et de l'habitat, 
de l'agriculture et de l'élevage (RGPHAE), Région de Dakar, $86 \mathrm{p}$.

[2] ANSD $^{\mathbf{b}}, 2017$ : Enquête nationale sur l'emploi au Sénégal, Quatrième trimestre 2016, Notes d'informations, $9 \mathrm{p}$.

[3] ANSD, 2013, Deuxième enquête de suivi de la pauvreté au Sénégal (ESPS-II-2011), $191 \mathrm{p}$.

[4] Aubry C, 2013 : L'agriculture urbaine, Contributrice des stratégies alimentaires des mégapoles? Daniel Thevenot. 24 èmes Journées Scientifiques de l'Environnement - La transition écologique des mégapoles, Créteil, France. JSE-2013 (5).

[5] Aubry et al., 2010 : Fonction Alimentaire de l'agriculture urbaine au Nord et au Sud: Permanence et renouvellement des questions de recherche. Emilie COUDEL, Hubert DEVAUTOUR, Christophe Toussaint SOULARD, Bernard HUBERT. ISDA 2010, Jun 2010, Montpellier, France. Cirad-InraSupAgro, $13 \mathrm{p}$.

[6] BA, Abou et al., 2016 : Impacts sur la santé des pratiques des agriculteurs urbains à Dakar (Sénégal). In: VertigO, vol. 16, n ${ }^{\circ} 1,16 \mathrm{p}$.

[7] Ba A., 2008, "L'agriculture de Dakar : quelle multifonctionnalité et quelles perspectives ? ». In Vidal R (dir.) : La diversité de l'agriculture urbaine dans le monde, vol. 3 des actes du colloque Les agricultures périurbaines, un enjeu pour la ville. () ENSP, Université de Nanterre, $12 \mathrm{p}$.

[8] Ba A et Aubry C., 2011: «Diversité et durabilité de l'agriculture urbaine : une nécessaire adaptation des concepts ? », Norois [En ligne], 221 |, mis en ligne le 30 décembre 2013, consulté le 30 septembre 2016. URL : http://norois.revues.org/3739 ; DOI : 10.4000/norois.3739.

[9] Bricas N., Seck P-A., 2004 : L'alimentation des villes du Sud : les raisons de craindre et d'espérer, Cahiers Agriculture, $\mathrm{n}^{\circ}$ 13, pp10-14.

[10] Bricas et al., 2003 : Sécurité alimentaire et urbanisation : enjeux pour l'agriculture intra et périurbaine. In Séance de l'Académie d'Agriculture de France Approvisionnement vivrier des villes du Sud : les enjeux et questions d'une agriculture de proximité, Paris, France, 12p.

[11] Casale K. 2006 : « Jardins démonstratif à Almirante Brown en Argentine ». In Multifonctionnalité de l'agriculture urbaine, Magazine Agriculture urbaine $\mathrm{n}^{\circ} 15,3$ pages.

[12] Chagomoka et al., 2015: Contribution of urban and periurban agriculture to household food and nutrition security in Ouagadougou, Burkina Faso. In: Renewable Agriculture and Food Systems FirstView, pp 1-16
[13] Kedowide, C. et al., 2010 : Dynamique spatiotemporelle de l'agriculture urbaine à Ouagadougou : Cas du Maraîchage comme une activité montante de stratégie de survie. In VertigO, Vol $10 \mathrm{n}^{\circ} 2,22 \mathrm{p}$.

[14] Dabat et al., 2012 : Le cresson à Antananarivo (Madagascar) : entre intérêts alimentaires et risques. In : Le Courrier de l'Environnement de l'INRA, (69), pp 99-112.

[15] Danso et al., 2002: Income of farming systems around Kumasi. Urban Agriculture Magazine, 7, p. 56.

[16] De Bon et al., 2010: Sustainable urban agriculture in developing countries. A review. In: Agronomy for Sustainable Development, vol. 30, ${ }^{\circ} 1$, pp. 21-32.

[17] Dubbeling et al., 2010: Cities, poverty and food: multi-stakeholder policy and planning in urban agriculture. RUAF, Practical Action Publishing, 189 p.

[18] Duchemin E., 2013 : Multifonctionnalité de l'agriculture urbaine : perspective de chercheurs et de jardiniers. In : Agriculture urbaine : aménager et nourrir la ville, Éd : Vertigo, pp. 97-107.

[19] Duchemin et al., 2008. Urban agriculture: multidimensional tools for social development in poor neighbourhoods », Field Actions Science Reports [Online], Vol. 1, Online since 28 November 2008, connection on 18 May 2015. URL : http://factsreports.revues.org/113.

[20] Diagne, A B. 2008, La problématique du maraîchage dans la communauté rurale de Sangalkam, département de Rufisque. Mémoire de DEA Département de géographie, Université Cheikh Anta Diop, Dakar.100 p.

[21] Gaye, M., et S. Niang, 2010, Manuel des bonnes pratiques d'utilisation saine des eaux usées dans l'agriculture urbaine, Dakar, ENDA-RUP, 126 p.

[22] Golhor, K., 1995 : L'agriculture urbaine en Afrique tropicale : évaluation in situ pour initiative régionale. In : Cities Feeding People Series, Report 14, 27 p.

[23] GRDR, 2015 : Le système alimentaire de la région de Dakar : État des lieux et perspectives face aux enjeux du changement climatique, $44 \mathrm{p}$.

[24] Guèye-Girardet, A, 2010, Évaluation des pratiques d'irrigation, de fertilisation et d'application des pesticides dans l'agriculture périurbaine de Dakar, Sénégal, Thèse de doctorat en Géosciences de l'université de Lausanne, $192 \mathrm{p}$.

[25] Guèye el al., 2009: Agriculteurs dans les villes ouestafricaines: Enjeux fonciers et accès à l'eau (IAGU, KARTHALA, CREPOS.) Dakar, 191p.

[26] IAGU, Diédhiou, 2011 : Le maraîchage urbain à Lendeng (Rufisque, Sénégal), Rapport d'étude, 73 p. 
[27] Maxwell, D., 2003: The importance of urban agriculture to food and nutrition. In: Annotated Bibliography on Urban Agriculture. SIDA and ETC, Leusden, The Netherlands, pp.22-129.

[28] Mbaye et Moustier, 2000: Market-oriented urban agricultural production in Dakar. In: Growing cities, growing food: Urban agriculture on the policy agenda. Feldafing (Allemagne):

[29] DES pp. 235-256.

[30] Mfoukou-Ntsakala et al., 2006 : Agriculture urbaine et subsistance des ménages dans une zone de postconflit en Afrique centrale. In : Biotechnologie, Agronomie, Société et Environnement, Vol 10 vol. $30, n^{\circ} 3$, pp 237-249.

[31] Mougeot J. L. A, 2000 : Autosuffisance alimentaire dans les villes : l'agriculture urbaine dans les pays du Sud à l'ère de la mondialisation. In : Armer les villes contre la faim, CRDI, pp 11-26.

[32] Moustier et Fall., 2004 : Les dynamiques de l'agriculture urbaine : caractérisation et évaluation. In : Développement durable de l'agriculture urbaine en Afrique francophone : enjeux, concepts et métyhodes/Smith Olanrewaju B. (ed.), Moustier Paule (ed.), Mougeot Luc J.A. (ed.), Fall Abdou (ed.), Montpellier : CIRAD, p. 2343.

[33] Moutier et Pages, 1997 : Le périurbain en Afrique : une agriculture en marge ?, Économie rurale, $\mathrm{n}^{\circ} 241$, pp. 48-55.

[34] Mendret V., 2006 : L'agriculture périurbaine au risque de la ville ? Le cas de Ndiamniadio (Dakar, Sénégal), mémoire de Master 1 en géographie humaine, Université Louis Pasteur Strasbourg 1.

[35] N'Diénor M, 2014 : Recyclage des déchets urbains solides par l'agriculture urbaine à Dakar (Sénégal). In : POUR, vol.4 $n^{\circ} 224$, pp. 299-314.

[36] Niang et al., 2012 : Le maraîchage autour de la décharge de Mbeubeuss. In : Les décharges d'ordures en Afrique : Mbeubeuss à Dakar au Sénégal, IAGUKarthala., pp. 306-320.

[37] Niang A. K. M, 2014, Marâ̂chage péri-urbain à Dakar : dynamique spatio-temporelle, contraintes et stratégies d'adaptation dans la Grande Niaye de Pikine. Mémoire de maîtrise, Centre régional AGRHYMET, $92 \mathrm{p}$.

[38] Olahan A. 2010: Agriculture urbaine et stratégies de survie des ménages pauvres dans le complexe spatial du district d'Abidjan. In VertigO, vol.10 n 2, 16 p.

[39] Padilla M, 2004 : Approvisionnement alimentaire des villes Méditerranéennes et Agriculture Urbaine. In : Interfaces : agricultures et villes à l'Est et au Sud de la Méditerranée. J. Nasr et M. Padilla (Eds), Delta/Ifpo, pp. 79-94.
[40] Souré M et al., 2012 : Rapport du Sénégal sur l'utilisation saines des eaux usées en agriculture. État du Sénégal, 22 p.

[41] Soulard et Aubry., 2011 : Quelle utilisation de l'espace en zones rurales et périurbaines? Cultiver les milieux habités. In : Agronomie, environnement \& sociétés, vol.1, $\mathrm{n}^{\circ}$ 2,pp. 87-102.

[42] Sposito T, 2010 : Agriculture urbaine et périurbaine pour la sécurité alimentaire en Afrique de l'ouest: Le cas des micro-jardins dans la municipalité de Dakar, Thèse de doctorat de l'Università Degli Studi di Milano, $220 \mathrm{p}$.

[43] Stewart et al., 2013: What are the impacts of urban agriculture programs on food security in low and middle-income countries? In: Environmental Evidence, vol.3. $\mathrm{n}^{\circ} 7, \quad 13 \quad \mathrm{p}$. https://doi.org/10.1186/2047-2382-2-7

[44] Temple et Moustier, 2004 : Les fonctions et contraintes de l'agriculture périurbaine de quelques villes africaines (Yaoundé, Cotonou, Dakar). In : Cahiers d'études et de recherches francophones/ Agricultures, vol. 13, n ${ }^{\circ}$ 1, pp. 15-22.

[45] Toullalan M., 2012 : Les enjeux de la production et de l'approvisionnement alimentaires en Ile-deFrance. Rapport au CERS, Commission de l'agriculture, de l'environnement et de la ruralité, mars 2012, $138 \mathrm{p}$.

[46] Tounkara S., 2015: La valorisation des déchets organiques dans l'agriculture «péri-urbaine » à Dakar (Sénégal). Analyse d'une multifonctionnalité stratégique. Thèse en sociologie, Université de Toulouse Jean Jaurès, $458 \mathrm{p}$.

[47] United Nations Population Fund (UNFPA), 2016 : 10 ans : comment cet âge déterminant chez les filles détermine notre avenir? Document technique, New York, $116 \mathrm{p}$.

[48] Véron J., 2007 : La moitié de la population mondiale vit en ville. In Population \& Sociétés $n^{\circ} 435$, juin 2007- Bulletin mensuel d'information de l'Institut national d'études démographiques.

[49] Wade, I et Lançon, F., 2015: Urbanisation, changements des habitudes alimentaires et transformations rurales en Afrique de l'Ouest. In Global Development Network Conference.

[50] Zezza et Tasciotti, 2010: Urban agriculture, poverty and food security: Empirical evidence from a sample of developing countries, Food policy, $\mathrm{n}^{\circ} 35$, pp. 265 273. DOI : $10.1016 /$ j.foodpol.2010.04.007. 